Published by Al-Nahrain College of Medicine P-ISSN 1681-6579

E-ISSN 2224-4719

Email: iraqijms@colmed-alnahrain.edu.iq http://www.colmed-alnahrain.edu.iq http://www.iraqijms.net

\title{
Renal Biopsy Practice in Iraq: A Systematic Review
}

\author{
Ala Sh. Ali $^{1}$ FIBMS (Nephrology), Ali J. Al-Saedi ${ }^{2}$ FIBMS (Nephrology) \\ ${ }^{1}$ Nephrology and Renal Transplantation Centre, The Medical City, Baghdad, Iraq, ${ }^{2}$ Dept. of Medicine, College of \\ Medicine University of Baghdad, Baghdad, Iraq
}

\begin{abstract}
Background Renal biopsy is an indispensable tool for the accurate diagnosis and treatment of different forms of renal disease. Many advances have improved the utility and safety of renal biopsy procedure.

Objective To determine the extent to which renal biopsy used and how much it affects the Iraqi nephrology practice.

Methods A systematic review of articles published from 1990 to 2014 was carried out by searching Medline and Google scholar. We included all studies that concerned with renal biopsy as the main intervention. Eligible studies determined by predefined criteria were reviewed. Data from these studies combined and analyzed.

Results The search yielded 55 title, of which, eleven studies met the inclusion criteria. Analysis of the 11 studies (2278 patients) that underwent 2667 renal biopsies. Ten studies centered on native renal pathology and one study focused on allograft pathology.

Conclusion The Iraqi renal biopsy practice and pathology interpretation need to be rejuvenated by modern pathology services, data registry, and well-designed studies with clinical impact.

Keywords Renal biopsy, Allograft, Pathology, Iraq.

DOI: 10.22578/IJMS.14.4.13
\end{abstract}

List of abbreviation: $\mathrm{CKD}=$ Chronic kidney disease, $\mathrm{IF}=$ Immunofluorescence, $E M=$ Electron microscopy, $L M=$ Light microscopy, IHC = Immunohistochemistry, IP = Immune peroxidase, FSGS = Focal segmental glomerulosclerosis, $M C D=$ Minimal change disease, $M P G N=$ Membranoproliferative glomerulonephritis, IgAN = Immunoglobulin A nephropathy, LN = Lupus nephritis.

\section{Introduction}

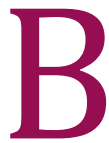

iopsy first defined by Besnier in 1895 became useful towards the end of nineteenth century ${ }^{(1)}$. Nils Alwall of Sweden performed the first systematic aspiration needle biopsies of the kidney in 1944 but the first published results were in 1951 by Iversen and Burn in Copenhagen. With the development of good histology, and the sequential appearance of new techniques like immunofluorescence (IF) (in 1956) and electron microscopy (EM) (in 1957), it became an integral part of clinical nephrology practice ${ }^{(1,2)}$.
Percutaneous renal biopsy is a relatively safe procedure and a powerful tool in the diagnosis and management of renal diseases (3). Refinement of biopsy technique and interpretation skills led to major development and a paradigm shift toward incorporation of modern molecular techniques. This was due to the explosion in kidney disease research in the last 20-30 years that brought new knowledge from bench to bedside and resulted in new molecular and genetic tools that enhance the diagnostic and prognostic power of the renal biopsy. These genomic technologies show a useful adjunct to the renal biopsy, and provide examples of how these may transform pathologic interpretation into molecular disease phenotypes ${ }^{(4)}$. 
Although most nephrologists recognize several clear indications for a renal biopsy, it is still underutilized ${ }^{(5)}$.

Organizing the multiplicity of information available in a renal biopsy to maximize benefits to the patient, as well as to the epidemiologist and researcher, is one of the challenges that face the nephrology community. In Iraq, renal biopsy practice started at late seventies with Menghini-type needles used for liver biopsy. Then tru-cut type needles used till now with very limited use of biopsy guns. Currently all renal biopsies are performed with the help of ultrasound $^{(6)}$.

The aim is to review the practice of these biopsies from indications, through technique to therapeutic and prognostic implications and to provide the best available evidence about how to fully utilize renal biopsy in Iraqi nephrology practice.

\section{Methods}

It is a systematic review using the PICO (Population, Intervention, Comparison, and Outcome) model ${ }^{(7)}$. Studies populations are patients (adults and children) who had been underwent renal biopsy. Inclusion criteria included: original articles publications, whether electronically or hard copies papers which are published in English language throughout the years from 1990 to 2014 and labeled as Iraqi studies. All these studies must incorporated renal biopsy as the main intervention or part of study protocol. While, the grey literatures and unpublished data were not considered.

Searching keywords included (renal biopsy, renal pathology, glomerular, glomerulonephritis and Iraq,). Different combinations of the above keyword might also help in searching and truncation method used. Search was through full text articles and abstracts ${ }^{(8)}$.

\section{Results}

The first hit of data bases was (55), then with the use of exclusion criteria (and) in combining the different keyword searches we reached to (39) search results. After removal of duplicates only (14) remained. With the use of other inclusion criteria only (11) articles left. All of them are original studies (Figure 1).

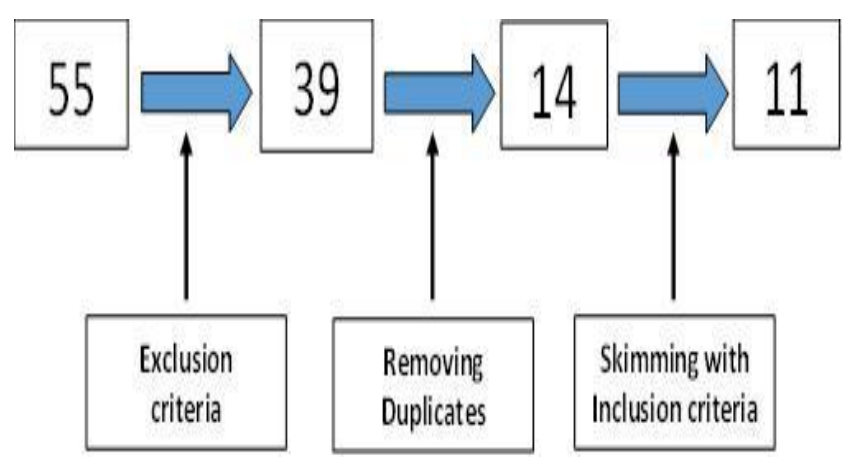

Figure 1. Data Collection

The total number of subjects included in these eleven papers is 2278. Eleven original articles have been reviewed. Ten studies involved adult patients and only one study was among pediatric age group. One study focused on allograft biopsy while all the other nine were studies of native renal pathology. A list of the eleven studies is given in table 1 , representing the name of authors, year of publication, the corresponding journal, type of study, number of participant, and study objectives. The results of searched articles will be presented under the following headings: Study objectives, Practice and technique, Pathology and Therapeutic and prognostic implications.

\section{Study Objectives}

By reviewing the above studies, we found that there is more or less an agreement between almost all the studies about the main objective of the studies which is the spectrum of glomerular disease. Seven out of eleven studies conducted to have a final conclusion on glomerular diseases pattern whether by light microscopy (LM) or IF. The study by Al-Taee ${ }^{(9)}$ 
presents the spectrum of amyloidosis causes in Iraq. The other three studies aimed clinicopathologic correlates with therapeutic and prognostic impacts ${ }^{(10,11,12)}$.

The study by Al-Omairi seems to be a study for correlation between pathology and therapeutic response in childhood lupus ${ }^{(13)}$. This in turn dictated the design of these studies. Eight of them were retrospective and cross sectional studies. The other two were prospective studies to show the effect on prognosis or to describe the effect of therapeutic intervention $(11,12)$

\section{Practice and Technique}

Two studies described the biopsy procedure $(12,14)$. Menghini-type needle used in most of biopsies before 2005. In the later years, semiautomated needle was the main tool. Studies by $\mathrm{Ali}^{(12)}$ and Al-Saedi ${ }^{(14)}$ described the use of different gauge needles, 16 and 18 gauge. Also, these two studies described the use of ultrasound either in pre-biopsy marking or in real time biopsy guidance. One study described the rate of complication and safety of biopsy ${ }^{(12,14)}$. There was very limited data about the rate of redo or serial biopsies.

\section{Pathology}

Light microscopy was the standard modality of nephropathology in all studies. The oldest study was by Salim and Ezzat ${ }^{(6)}$ showed the full renal pathology lab potential through the study of 478 biopsies. All specimens were studied by LM and a small number, when available; had been proceeded for IF and EM. Unfortunately, neither IF, nor EM are not available for histopathology services for the last twenty years. Two studies described the practice of cutting, fixation and staining for all steps of biopsy sample examination ${ }^{(6,12)}$. One study entirely based on IF ${ }^{(15)}$ and this had been conducted through collaboration with a pathology lab outside Iraq. IF findings also described in allograft biopsy by immunohistochemistry using immune peroxidase (IP) ${ }^{(12)}$. Data about adequacy of
Iraqi renal biopsies recorded in two studies only $(12,16)$. Still focal segmental glomerulosclerosis (FSGS) is the most common primary glomerular pathology in Iraqi biopsy specimens. Together with minimal change disease (MCD) they consist about $45-50 \%$. This was evident in four studies ${ }^{(11,14,17,18)}$. Even with IF, FSGS was the most prevalent lesion ${ }^{(15)}$. In one study by Al-Saedi, membranoproliferative glomerulonephritis was the most prevalent ${ }^{(16)}$. Tuberculosis is the most common cause of renal amyloidosis in Iraq ${ }^{(9)}$. Salim and Ezzat reported renal involvement in systemic disease in $14 \%$ of the examined biopsies ${ }^{(6)}$.

\section{Therapeutic and Prognostic Implications}

Two studies described clinicopathological correlates $(10,11)$. One study showed the prognostic value of tubulointerstitial lesion in renal biopsies for patients with glomerular disorders ${ }^{(11)}$. One study disclosed the role of biopsy in guiding therapeutic intervention ${ }^{(12)}$.

\section{Discussion}

This systematic review included 2667 renal biopsies and showed a major finding that the main objectives of Iraqi renal biopsy studies was just to describe the pattern of their findings on individual bases of their respective renal units. In addition, it's apparent that all this practice faces challenges that need to be addressed. It's well known how much renal pathology is essential for nephrology practice but in this study, we tried to have evidence how much Iraqi practice of renal biopsy influenced the care for Iraqi renal patients.

Study design: is the first challenge in renal biopsy studies. It would be dictated always by the question why we need renal pathology based studies. Do we need just shallow data or we need in depth studies that full-fill the terms of validity and usefulness? According to this systematic review still there is no consensus Iraqi nephrology practice about indications, the procedure, interpretation, clinicopathological correlates, complications, and prognostic implications. 
Many developed countries have established national renal biopsy registries to document such variations and changing trends in the disease spectrum. However, such registries are largely lacking or are in primitive shape in most of the developing countries ${ }^{(19)}$.

Table 1. Studies included in the review by the date of publication

\begin{tabular}{|c|c|c|c|c|c|}
\hline Author & Year & Journal & $\begin{array}{l}\text { Patients } \\
\text { No. }\end{array}$ & $\begin{array}{l}\text { Type of } \\
\text { study }\end{array}$ & Aim and Results \\
\hline $\begin{array}{l}\text { Salim and } \\
\text { Ezzat }\end{array}$ & 1997 & J Fac Med Baghdad & 369 & Original & Pattern of Glomerular Ds \\
\hline Shaker et $\mathrm{al}^{17}$ & 2002 & SJKDT & 520 & Original & Pattern of Glomerular Ds \\
\hline $\begin{array}{l}\text { Al-Duliemi et } \\
\text { al }^{10}\end{array}$ & 2005 & J Fac Med Baghdad & 100 & Original & $\begin{array}{c}\text { Clinicopathological } \\
\text { Correlate }\end{array}$ \\
\hline $\begin{array}{l}\text { Mansour and } \\
\text { Al-Shamma }{ }^{18}\end{array}$ & 2005 & J ABMS & 136 & Original & Pattern of Glomerular Ds \\
\hline Al-Taee et al ${ }^{9}$ & 2005 & IPMJ & 1051 & Original & $\begin{array}{c}\text { Causes of renal } \\
\text { Amyloidosis }\end{array}$ \\
\hline $\begin{array}{l}\text { Mansour and } \\
\text { Al-Shamma }\end{array}$ & 2006 & Med Gen Med & 136 & Original & Prognostic implications \\
\hline Al-Saedi ${ }^{16}$ & 2009 & NIJM & 80 & Original & Pattern of Glomerular Ds \\
\hline Al-Saedi et al ${ }^{14}$ & 2011 & Al-Kindy Col Med J & 120 & Original & Pattern of Glomerular Ds \\
\hline Ali et al $^{12}$ & 2013 & SJKDT & 47 & Original & Allograft Biopsy \\
\hline $\begin{array}{l}\text { Al-Saegh and } \\
\text { Assad }{ }^{15}\end{array}$ & 2013 & AJNT & 58 & Original & $\begin{array}{c}\text { IF pattern of Glomerular } \\
\text { Ds }\end{array}$ \\
\hline Al-Omairi ${ }^{13}$ & 2014 & AJNT & 50 & Original & $\begin{array}{l}\text { Glomerular pattern in } \\
\text { childhood SLE }\end{array}$ \\
\hline
\end{tabular}

This gap is partially filled by single or multicenter data on renal biopsies in some of these countries. Although these are not ideal, hospital or center-based biopsy studies do shed some light on the spectrum of the prevalent renal diseases in that particular location ${ }^{(19)}$. Thus, these partial results cannot conclude that the present sample of patients (2278) is actually representative of the nationwide frequency of glomerular, tubulointerstitial, or renal vascular diseases or renal grafts in Iraq.

Registry data from Saudi Arabia showed the distribution and epidemiology of primary and secondary glomerular diseases. Italian experience of national renal biopsy registry concluded how much this results meet the current challenges facing the clinical research enterprise ${ }^{(20-22)}$. The population included are children, adults, and elderly. The registry can provide separate analysis of different groups and would have been more informative and reflective of the demographics. In addition, renal allograft biopsy still not widely practiced, not well documented, and its utility is affected by many logistic issues. There are at least 500 transplants annually in Iraq and the number of graft biopsies is not concordant with these increasing number of transplants. By this we are unable to explain acute and graft dysfunction and this certainly will affect 
management plan. Still there is a low threshold for graft biopsy especially early post-transplant mainly because of risk of bleeding that should be weighed against the possibility of losing a graft because of an unknown diagnosis ${ }^{(12,23)}$.

The identified studies disclosed that the practice of renal biopsy as a procedure merits reevaluation. There was no adequate data about adequacy of the biopsy sample and also how much the needle gauge size affects it. Specimen adequacy assessment is the initial step during the assessment of a native renal and allograft samples. Specimen adequacy is one of the determinant of diagnostic agreement in the histopathological assessment as it should be considered in the view of clinical data. Adequacy statement in the renal biopsy report and on-site adequacy assessment should be a standard approach ${ }^{(24,25)}$. Light microscopy is the main tool for biopsy interpretation. Immunohistochemistry (IHC) but not IF is the standard in the current Iraqi renal pathology services. The sensitivity, specificity of IHC on formalin-fixed paraffinembedded renal biopsy specimens is lower than IF with some drawbacks and therefore, not always suitable for evaluation of renal biopsies ${ }^{(26)}$.

Complement antigens require careful, timeconsuming antigen-retrieval procedures because of antigen masking during processing into paraffin. A false negative can be a concern. Interpretation of IHC slides is also complicated by potentially higher background in renal tissues and the difficulty of titrating the final color product to avoid over or under staining. Also, since the IP signal in IHC is a reaction product dependent on interaction of substrate and enzyme, diffusion artefacts can create problems in interpretation ${ }^{(26,27)}$. The cause behind using IHC in Iraqi studies is the unavailability of proper renal pathology lab, unavailability of IF microscopy, no proper nephropathology training, and the need for sample transport across long distance to be interpreted. In addition, EM is not available in our current practice and again this will add more challenges ${ }^{(27)}$. Most of the studies in this review didn't provide data about the complications of biopsy procedure and if there is any intervention to prevent and/or to correct. This should be in the context of improved techniques using ultrasound guidance for percutaneous renal biopsy ${ }^{(28,29)}$. A major finding of the studies is that FSGS is the commonest cause of primary nephrotic syndrome (NS). Still there is no data about classification of FSGS into histological variants. In one study, membraoproliferative glomerulonephritis was the most common cause but this needs to be critically appraised in the view of modern classification of MPGN. Another common finding in these studies is that IgA nephropathy (IgAN) is not the most prevalent, although it is considered the most common primary glomerular disease worldwide ${ }^{(30)}$. In the Middle East, FSGS is the most frequent renal disease as in the Saudi Arabian Registry, followed by MPGN. IgAN accounts for only $6.5 \%$, while $\mathrm{LN}$ is the most common secondary form $(20,22)$. These histopathological lesions of glomerular diseases observed on renal biopsies need to be correlated with different clinical and demographic features of the patients. This in turn represents how much the indication of biopsy need to be documented and not restricted to glomerular disease diagnosis and to include other justifiable clinical indication like unexplained acute kidney injury, interstitial nephritis, or renal involvement by systemic disease. A renal biopsy doesn't only help to establish and confirm a suspected diagnosis, it may aid in understanding the mechanism of disease. It also provides useful prognostic information on the degree of glomerular, vascular and tubulointerstitial involvements and can also help in guiding treatment plan, determining the effectiveness of recommended therapy, or when treatment is futile ${ }^{(4,30)}$. Apart from few studies Iraqi renal biopsy lack these endpoints. This may be related to study design, and lack of proper follow up. This systematic review had some 
limitations include the limited search strategy with the publications and sites where these articles had been published. Possibly they were non-homogenous in terms of study designs, sample size or endpoints. They were not randomized controlled trials but of acceptable quality.

In conclusion; Iraqi practice of renal biopsy needs to be extended to full spectrum to have the full benefits of renal pathology. This can be achieved through proper and modern pathology laboratory, dedicated renal pathology training and adoption of modern renal histopathology approaches. Creating an environment for a precise documentation of all clinical and pathology data in all renal units will make the bases for national renal pathology registry. The data about the spectrum of glomerular disease should be translated in to this national registry for all renal units over fair follow up period to have the exact statistics and to put the rationale for management, follow up, and future detailed analysis. Renal biopsy data should be the result of well-designed studies that hit the depth of renal pathology for better understanding of disease mechanism, induction of targeted therapy and prognostic predictions.

\section{Acknowledgement:}

None.

\section{Author Contribution}

Dr. Ali: Design, Protocol preparation and manuscript writing. Dr. Al-Saedi: data search, collection and literature review.

\section{Conflict of Interest}

Nothing to disclose.

\section{Funding}

Authors received no funds to complete this study apart from self-funding.

\section{References}

1. Iversen $P$, Brun C. Aspiration biopsy of the kidney. Am J Med. 1951; 11: 324-30.

2. Cameron JD, Hicks J. The introduction of renal biopsy into nephrology from 1901 to 1961: a paradigm of the forming of nephrology by technology. Am J Nephrol. 1997; 17: 347-58.

3. Nelson CL, Mackinnon MWB, Charlesworth JA. Importance of renal biopsy. Nephrology. 2001; 6: 270-3.

4. Dhaun N, Bellamy CO, Cattran DC, et al. Utility of renal biopsy in the clinical management of renal disease. Kidney Int. 2014; 85: 1039-48.

5. Liapis $H$, Gaut JP. The renal biopsy in the genomic era. Pediatr Nephrol. 2013; 28: 1207-19.

6. Salim MS, Ezzat WR. Renal histopathological lesions in Iraq with particular reference to glomerulonephritis. J Fac Med Baghdad. 1997; 39: 352-8.

7. Sackett DL, Richardson WS, Rosenberg W, et al. Evidence-based medicine: How to practice and teach EBM. 2nd Ed. New York: Churchill Livingston; 2000.

8. Treadwell JR, Singh S, Talati R, et al. A Framework for "Best Evidence" Approaches in systematic reviews [Internet]. Rockville (MD): Agency for Healthcare Research and Quality (US); 2011 Jun. Methods/Approaches. Available from: https://www.ncbi.nlm.nih.gov/books/NBK56660/.

9. Al-Taee K, Al-Mukhtar S, Al-Shamma I. Causes of renal amyloidosis in Iraq. Iraqi Postgrad Med J. 2005; 4: 268-72.

10. Al-Duliemi KK, AL-Saedi AJH, Saleem MS, et al. Clinicopathological correlation in 100 patients with membranous nephropathy. J Fac Med Baghdad. 2005; 47: 343-6.

11. Mansour AA, Al-Shamma I. Significance of tubulointerstitial lesions in kidney biopsy specimen of nephrotic patients in Iraq. Medscape General Medicine 2006; 8(1): 8.

12. Ali AA, Al-Mudhaffer AJM, Al-Taee $Q$, et al. Allograft biopsy in kidney transplant recipients in the medical city of Baghdad. Saudi J Kidney Dis Transpl. 2013; 24: 1039-43.

13. Al-Omairi OA. Clinical presentation and management outcome of childhood-onset systemic lupus erythamatosus in Baghdad. Arab J Nephrol Transplant. 2014; 7: 125-7.

14. Al-Saedi AJ, Abdul Mahdi M, Jameel NS. Results of kidney biopsies among adult Iraqi patients in a single center. Al-Kindy Col Med J 2011; 7: 82-4.

15. Al-Saegh RM, Assad LW. The Spectrum of glomerular diseases as studied by immunofluorescence microscopy: a single center study in Iraq. Arab J Nephrol Transplant. 2013; 6: 161-7. 
16. Al-Saedi AJ. The histopathological pattern of nondiabetic glomerular disease among adult diabetic patients. New Iraqi J Med. 2009; 5: 84-6.

17. Shaker Ikdam K, Al-Saedi Ali JH, Al-Salam Suhail, Saleem Mahassim S, Al-Shamma Ihsan A. Spectrum of Glomerular Disease in Iraqi Patients from a Single Center. Saudi J Kidney Dis Transpl. 2002; 4: 515-9.

18. Mansour AA, AL-Shamma IA. Primary glomerulonephritides causing nephrotic syndrome in young adults in Iraq. J Arab Board Med Special. 2005; 7: 302-4.

19. Mubarak M. Toward establishing a renal biopsy registry: A step in the right direction. Indian J Nephrol. 2013; 23: 159-60.

20. Huraib S, Al Khader A, Shaheen FA. et al. The spectrum of glomerulonephritis in Saudi Arabia: the results of the Saudi Registry. Saudi J Kidney Dis Transpl. 2000; 11: 434-41.

21. Gesualdo L, DI Palma AM, Morrone LF, et al. The Italian experience of the national registry of renal biopsies. Kidney Int. 2004; 66: 890-4.

22. Pesce F, Schena FP. Worldwide distribution of glomerular diseases: the role of renal biopsy registries. Nephrol Dial Transplant. 2010; 25: 334-6.

23. Ali AS, Al-Mallah $S$, Al-Saedi A. Renal transplantation in Iraq: history, current status, and future perspectives. Iraqi New Med J. 2016; 2(1): 10-14.

24. Cimen S, Geldenhuys L, Guler S, et al. Impact of specimen adequacy on the assessment of renal allograft biopsy specimens. Braz J Med Biol Res.
2016; 49(4): e5301. doi: 10.1590/1414431X20165301.

25. Geldenhuys L, Nicholson $P$, Sinha $N$, et al. Percutaneous native renal biopsy adequacy: a successful interdepartmental quality improvement activity. Can J Kidney Health Dis. 2015; 2: 8. doi: 10.1186/s40697-015-0043-z.

26. Rathore MU, Khadim MT, Mushtaq S, et al. Paraffinbased immunohistochemistry in the evaluation of glomerular diseases in renal biopsies. J Coll Phys Surg Pakistan. 2012; 22: 353-7.

27. Walker PD, Cavallo T, Bonsib SM, et al. Practice guidelines for the renal biopsy. Mod Pathol. 2004; 17: 1555-63.

28. Toledo K, Pérez MJ, Espinosa M, et al. Complications associated with percutaneous renal biopsy in Spain, 50 years later. Nefrologia. 2010; 30: 539-43.

29. Manno C, Strippoli GF, Arnesano L, et al. Predictors of bleeding complications in percutaneous ultrasoundguided renal biopsy. Kidney Int. 2004; 66(4): 1570-7.

30. AK Soyibo, EN Barton. Importance of a Renal Biopsy. West Indian Med J. 2009; 58: 270-2.

\section{Correspondence to Dr. Ala Sh. Ali E-mail: ala1975@gmail.com Received $30^{\text {th }}$ Mar. 2016: Accepted $27^{\text {th }}$ Nov. 2016}

